\title{
MULTISCALE MODELING OF RECRYSTALLIZATION
}

\author{
M.A.MIODOWNIK ${ }^{*}$, E.A.HOLM*, A.W.GODFREY ${ }^{* *}$, D.A. HUGHES ${ }^{* *}$, R. LESAR ${ }^{+}$. \\ "Sandia National Laboratories, Albuquerque, New Mexico. \\ ${ }^{* *}$ Sandia National Laboratories, Livermore, California. \\ ${ }^{+}$Los Alamos National Laboratory, Los Alamos, New Mexico.
}

\begin{abstract}
We propose a multi length scale approach to modeling recrystallization which links a dislocation model, a cell growth model and a macroscopic model. Although this methodology and linking framework will be applied to recrystallization, it is also applicable to other types of phase transformations in bulk and layered materials. Critical processes such as the dislocation structure evolution, nucleation, the evolution of crystal orientations into a preferred texture, and grain size evolution all operate at different length scales. In this paper we focus on incorporating experimental measurements of dislocation substructures, misorientation measurements of dislocation boundaries, and dislocation simulations into a mesoscopic model of cell growth. In particular, we show how feeding information from the dislocation model into the cell growth model can create realistic initial microstructures.
\end{abstract}

\section{INTRODUCTION}

The role of recrystallization models is to predict the influence of external variables such as strain, strain rate, and temperature on microstructure and texture. Macroscopic models characterize the microstructure in terms of state variables, such as dislocation content, cell size and texture [1]. These are related to the external variables via constitutive equations of recrystallization and recovery derived either analytically or empirically by fitting to experiment. Macroscopic models have achieved considerable success when applied systematically to specific systems of interest, such as multi-pass rolling of steels [2,3]. However they do not include any details about the microstructure of the alloy, except in terms of average properties. It is becoming increasing clear that heterogenities present in the microstructure greatly influence the nucleation process $[1,4]$.

Deformation microstructures consist of cellular patterns of dislocation boundaries. In so much that this is similar to a grain structure, it might seem that the Monte Carlo Potts model would be well suited to simulate evolution of such a structure. The Potts model is extremely successful in modeling annealing processes such as grain growth [5], and secondary recrystallization [6]. It has been applied to primary recrystallization by a number of workers, but in all cases nucleation has been imposed on the simulation either by directly inputing nuclei [7] or by imposing nucleation criteria [8]. In both cases the model is employed as a macroscopic model to investigate the effect of heterogeneous nucleation on the final microstructure and texture. These studies do not reveal information about the nucleation mechanism of recrystallization.

The central question is how do high angle recrystallization nuclei emerge from an evolving structure of dislocation boundaries? There is currently a great deal of experimental work characterizing dislocation substructures. Since it is not feasible to map out the deformation structure of a material, structural laws need to be developed through statistical sampling of small volumes using the TEM. This approach has been pioneered by Hughes et al.[9] who have proposed a scaling law for dislocation boundaries in medium to high stacking fault energy fcc metals. Such work is important because these laws can allow models of cell growth to incorporate realistic starting microstructures. Experimental work to elucidate the kinetic law for the motion of dislocation boundaries is also a vital piece of the jigsaw. Many workers have assumed that dislocation boundaries will migrate under curvature driven growth. It is not clear that this is valid; in particular one would expect dislocation climb and glide to greatly influence boundary migration. 


\section{DISCLAIMER}

Portions of this document may be illegible in electronic image products. Images are produced from the best available original document. 
We model recrystallization using a multiscale approach. A dislocation model is employed to examine the dynamics of dislocation cell structures and in particular to develop a kinetic law for dislocation boundary motion. This is fed into a mesoscopic simulation in which we model boundaries instead of dislocations, allowing us to model 3D microstructures and simulate a large number of cells. The initial boundary misorientation distribution (BMD) is compared to the experimental BMDs measured by Hughes et al [9]. Parameters such as nucleation rates and nuclei orientation are fed from the mesoscopic model into a macroscopic model of recrystallization.

In this short paper we describe briefly the dislocation model and the cell growth model, and show how feeding information between them enables us to develop realistic starting microstructures which compare well with experiment.

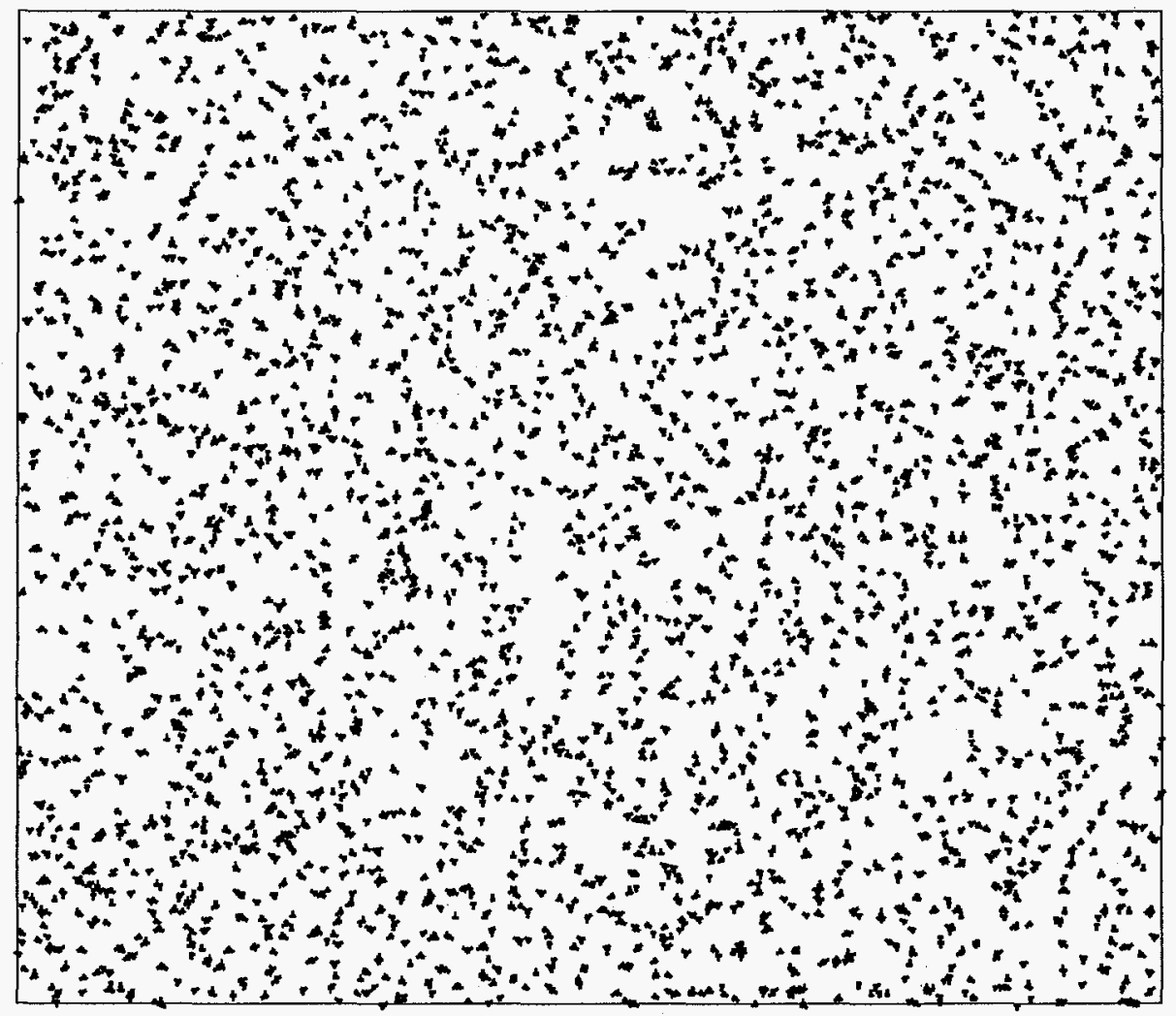

Figure 1. A relaxed dislocation structure with 5000 dislocations.

\section{DISLOCATION MODEL}

A 2D Monte Carlo dislocation model was used to create experimentally realistic dislocation cell structures. The simulation volume was discretized into a triangular lattice with periodic boundary conditions and Burger vectors along each principle direction. The initial configuration consisted of $\mathrm{N}$ randomly placed edge dislocations. The energy of the system was defined in terms of the sum of the potential between pairs of dislocations $(i, j)$ in the system. Using the stress tensor of a periodic array of dislocation $(j)$, the force on another dislocation $(i)$ can be calculated using the Peach-Kohler equation. The interaction potential between the dislocations is then given by [10]:

$$
\Phi_{i j}=\int\left(b_{i, x} \sigma_{j, x x}+b_{i, y} \sigma_{j, x y}\right) d y
$$

where $\sigma_{j, x x}$ and $\sigma_{j, x y}$ are components of the stress tensor of dislocation $(j)$ and $b_{i, x}$ and $b_{i, y}$ are components of the burgers vector of dislocation $(i)$ in the $x$ and $y$ directions. The energy, $E$, of the system is the sum of the interaction potentials. The system is relaxed using a Monte Carlo method. 
A dislocation is chosen at random and the change in energy, $\Delta E$, associated with a proposed move of unit distance along its slip direction is calculated. This move is accepted if $\Delta E<0$. At present the simulation does not include dislocation climb, dislocation reactions or annihilations, though these will be introduced imminently. Figure 1 shows the relaxed dislocation structure for a system with 5000 dislocations. There is little appearance of cell formation, though a large number of dislocation dipoles are formed.

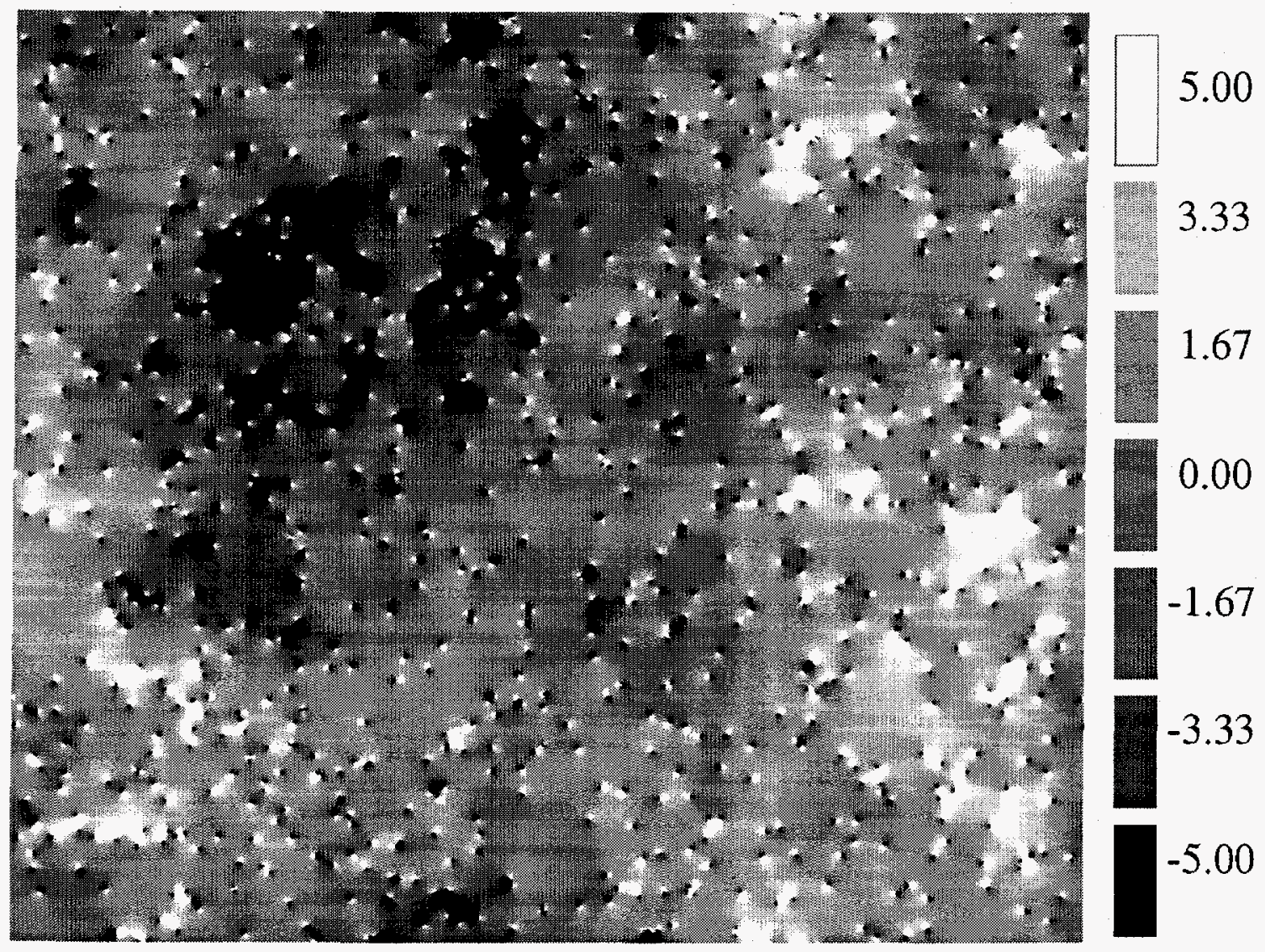

Figure 2. The skew-symmetric orientation map of the relaxed dislocation structure of figure 1.

A quantitative measure of the orientation of the lattice is given by the skew-symmetric rotation matrix:

$$
\theta_{x, y}=\frac{1}{2}\left(\frac{\partial u_{y}}{\partial x}-\frac{\partial u_{x}}{\partial y}\right)
$$

where $u_{y}$ and $u_{x}$ are the displacement components of each site $(x, y)$.

These orientations are plotted in figure 2. A degree of cell formation is evident, although it should be noted that the relaxed structure and random configuration (not shown) share the same general features. The rotation angle, $\Delta \theta$, of each cell is defined as, $\theta_{x, y}-\theta_{\text {ave }}$, where $\theta_{\text {ave }}$ is the average orientation (very close to zero degrees in this simulation). The probability function of $\Delta \theta$ is shown in figure 3 , showing that the relaxed dislocation structures have a Gaussian distribution of misorientations. We feed this result into the Cell Growth Model to generate initial microstructures. 


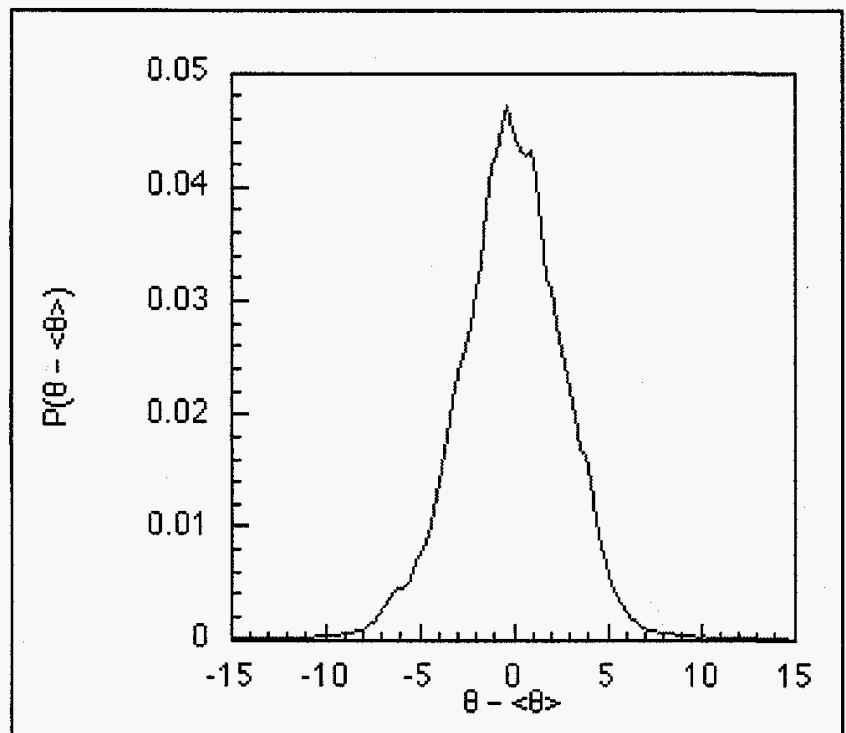

Figure 3. The distribution of rotation angles in the dislocation model

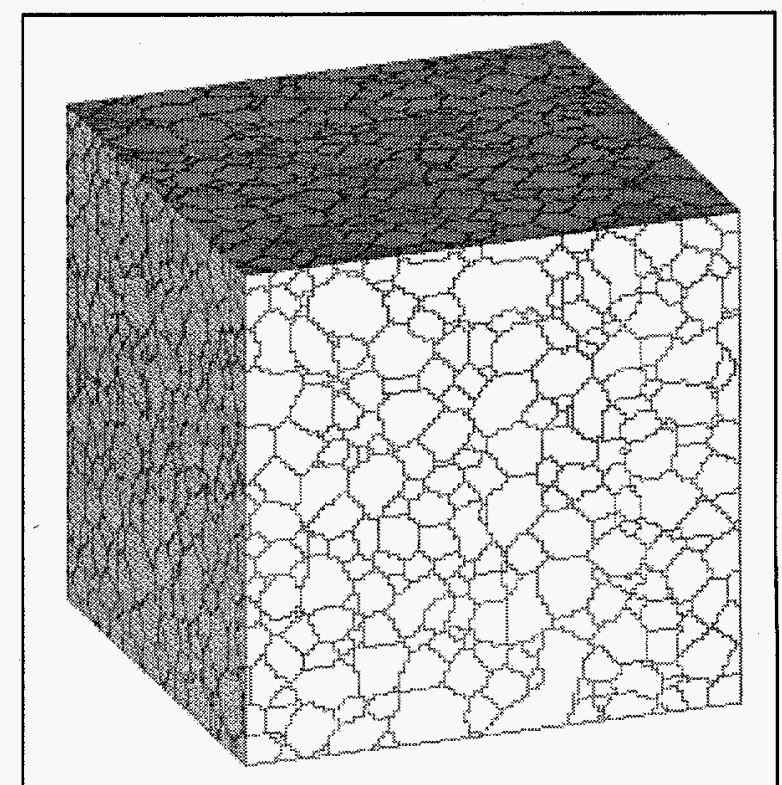

Figure 4. The equiaxed cell microstructure

\section{CELL GROWTH MODEL}

We use a modified Potts model to simulate cell growth of dislocation substructures. A simulation volume is discretized into a simple cubic lattice of $N_{x} x N_{y} x N_{z}$ sites. An equiaxed cell structure is used as a starting configuration, see figure 4 . Each site has a spin number $i$, where $i=1 \ldots N_{c}$ cells in the system. A set of rotation angles $m_{i}$ is defined which has a Gaussian distribution, and a set of axes $[u v w]_{i}$ are defined which are randomly distributed with a uniform probability on a unit sphere. The transformation matrix $\boldsymbol{R}_{i}$ of a rotation of $m_{i}$ about $[u v w]_{i}$ is calculated and the orientation of each cell, $\boldsymbol{O}_{i}$ is then given by:

$$
O_{i}=O_{r e f} R_{i}
$$

This procedure reproduces the distribution of orientations exhibited by the dislocation model, i.e. a Gaussian distribution with a mean of zero. Misorientations across cell boundaries are used to measure the BMD of the system which is shown in figure 5. The standard deviation of the Gaussian rotation angles, $\mathrm{m}_{\mathrm{i}}$, was found to determine the average cell boundary misorientation but did not influence the shape of the BMD which scales with average misorientation angle. At present no dynamical simulations have been carried out. Boundary migration will be implemented using a Monte Carlo Metropolis algorithm incorporating the kinetic law derived from the dislocation model.

\section{COMPARISON WITH EXPERIMENT}

Dislocations developed during monotonic plastic deformation of medium to high stacking fault energy fcc metals organize into boundaries that subdivide an original grain at two length scales [9]. The larger scale is defined by long, continuous dislocation boundaries called geometrically necessary boundaries (GNBs). Within the volumes defined by these boundaries lower angle incidental dislocation boundaries (IDBs) are formed. The BMDs of GNBs and IDBs each evolve differently as a function of strain. 
To develop nucleation criteria based upon local heterogeneities in the deformation microstructure, it is necessary to construct starting microstructures that contain both these boundary types (GNBs and IDBs), and where each type has the experimentally observed misoirentation distribution. Hughes et al. have shown that for a wide range of fcc metals, the misorientation distributions of the IDBs exhibit a scaling property, with the varying distributions collapsing to a single curve when the misorientation probability density distribution is scaled by the average misorientation [9] according to

with

$$
p\left(\theta, \theta_{a v}\right)=\theta_{a v} f\left(\theta / \theta_{a v}\right)
$$

$$
f(x)=\frac{\alpha^{\alpha}}{\Gamma(\alpha)} x^{\alpha-1} \exp (-\alpha x)
$$

where $\Gamma(\alpha)$ is the gamma function evaluated at argument $\alpha$ (the fitting parameter), with $\alpha=3$. Figure 5 shows the comparison between this curve and that constructed as a starting microstructure in the Cell Growth Model. The good correlation between the two is encouraging, however it should be noted that GNBs have not been included in the model and are likely to have an important influence on the development of recrystallization nuclei. This is the focus of our current efforts.

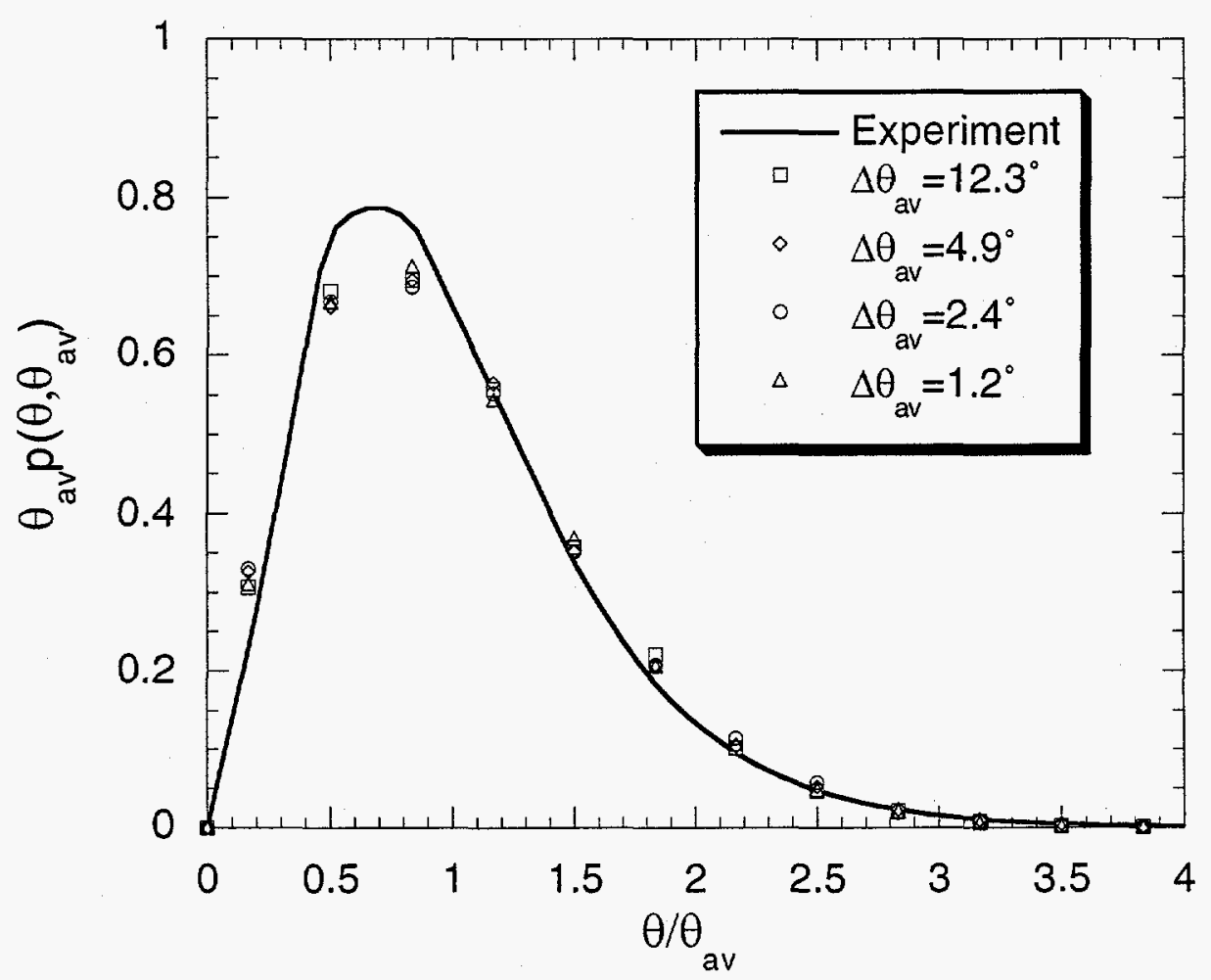

Figure 5. A Comparison of the BMD of the Cell Growth model with the experimentally fitted BMD 


\section{CONCLUSIONS}

We have modeled the formation of dislocation sub structures by linking a dislocation model to a cell growth model. The Gaussian spread of rotation angles which comes out of the dislocation model when applied the cell model produces a BMD that is very close to the experimental BMD of IDB boundaries. The next stage will be to incorporate dynamics into the dislocation model to measure a kinetic equation for dislocation boundaries. This will then be implemented into the Cell Growth model so that the behavior of large systems cells may be studied.

\section{ACKNOWLEDGMENTS}

This work was performed at Sandia National Laboratories, supported by the U.S. Department of Energy under contract number DE-AC04-94AL85000.

\section{REFERENCES}

1 F.J.Humphreys \& M.Hatherly, Recrystallization and Related Annealing Phenomena, (Pergamon Press, Oxford, 1996)

2. C.M.Sellars, (1990) Mats. Sci. and Tech. 6, p.1072.

3. C.M.Sellars \& J.A.Whiteman (1979) Met. Sci. 13, p.325.

4. J. Hjelen, R. Ørsund \& E.Nes (1991) Acta Metall. 39, p.1377.

5. Ling S. and Anderson M.P. (1992) JOM, Metals and Materials Society, TMS, Sept, p.30.

6. Srolovitz D.J., Grest G.S. and Anderson M.P. (1985), Acta Metall., 33, p.2233.

7. Srolovitz D.J., Grest G.S. and Anderson M.P. (1986), Acta Metall., 34, p.1833.

8. Holm and Battaile (private communication)

9. Hughes et al. (1997), Acta Metall., 45, p.105.

10. R.Lesar (private communication)

Sandia is a multiprogram laboratory operated by Sandia Corporation, a Lockheed Martin Company, for the United States Department of Energy under contract DE-AC04.94AL85000. 